\title{
A versatile photoelectron spectrometer for pressures up to 30 mbar
}

Susanna K. Eriksson, Maria Hahlin, Juhan Matthias Kahk, Ignacio J. Villar-Garcia, Matthew J. Webb, Helena Grennberg, Rositsa Yakimova, Hakan Rensmo, Kristina Edstrom, Anders Hagfeldt, Hans Siegbahn, Marten O. M. Edwards, Patrik G. Karlsson, Klas Backlund, John Ahlund and David J. Payne

\section{Linköping University Post Print}

\section{Tweet}

N.B.: When citing this work, cite the original article.

Original Publication:

Susanna K. Eriksson, Maria Hahlin, Juhan Matthias Kahk, Ignacio J. Villar-Garcia, Matthew J. Webb, Helena Grennberg, Rositsa Yakimova, Hakan Rensmo, Kristina Edstrom, Anders Hagfeldt, Hans Siegbahn, Marten O. M. Edwards, Patrik G. Karlsson, Klas Backlund, John Ahlund and David J. Payne, A versatile photoelectron spectrometer for pressures up to 30 mbar, 2014, Review of Scientific Instruments, (85), 7, 075119.

http://dx.doi.org/10.1063/1.4890665

Copyright: American Institute of Physics (AIP) http://www.aip.org/

Postprint available at: Linköping University Electronic Press

http://urn.kb.se/resolve?urn=urn:nbn:se:liu:diva-110982 


\section{A|P|P $\mid \begin{aligned} & \text { Review of } \\ & \text { Scientific Instruments }\end{aligned}$}

\section{A versatile photoelectron spectrometer for pressures up to $\mathbf{3 0} \mathrm{mbar}$}

Susanna K. Eriksson, Maria Hahlin, Juhan Matthias Kahk, Ignacio J. Villar-Garcia, Matthew J. Webb, Helena Grennberg, Rositza Yakimova, Håkan Rensmo, Kristina Edström, Anders Hagfeldt, Hans Siegbahn, Mårten O. M. Edwards, Patrik G. Karlsson, Klas Backlund, John Åhlund, and David J. Payne

Citation: Review of Scientific Instruments 85, 075119 (2014); doi: 10.1063/1.4890665

View online: http://dx.doi.org/10.1063/1.4890665

View Table of Contents: http://scitation.aip.org/content/aip/journal/rsi/85/7?ver=pdfcov Published by the AIP Publishing

\section{Articles you may be interested in}

Angle-resolved environmental X-ray photoelectron spectroscopy: A new laboratory setup for photoemission studies at pressures up to 0.4 Torr

Rev. Sci. Instrum. 83, 093112 (2012); 10.1063/1.4754127

Note: A combined aerodynamic lens/ambient pressure x-ray photoelectron spectroscopy experiment for the onstream investigation of aerosol surfaces

Rev. Sci. Instrum. 81, 016106 (2010); 10.1063/1.3276714

New setup for in situ x-ray photoelectron spectroscopy from ultrahigh vacuum to $1 \mathrm{mbar}$

Rev. Sci. Instrum. 76, 014102 (2005); 10.1063/1.1824351

Feasibility tests of transmission $\mathrm{x}$-ray photoelectron emission microscopy of wet samples

Rev. Sci. Instrum. 71, 11 (2000); 10.1063/1.1150151

Performance of the grating-crystal monochromator of the ALOISA beamline at the Elettra Synchrotron

Rev. Sci. Instrum. 70, 3855 (1999); 10.1063/1.1150001

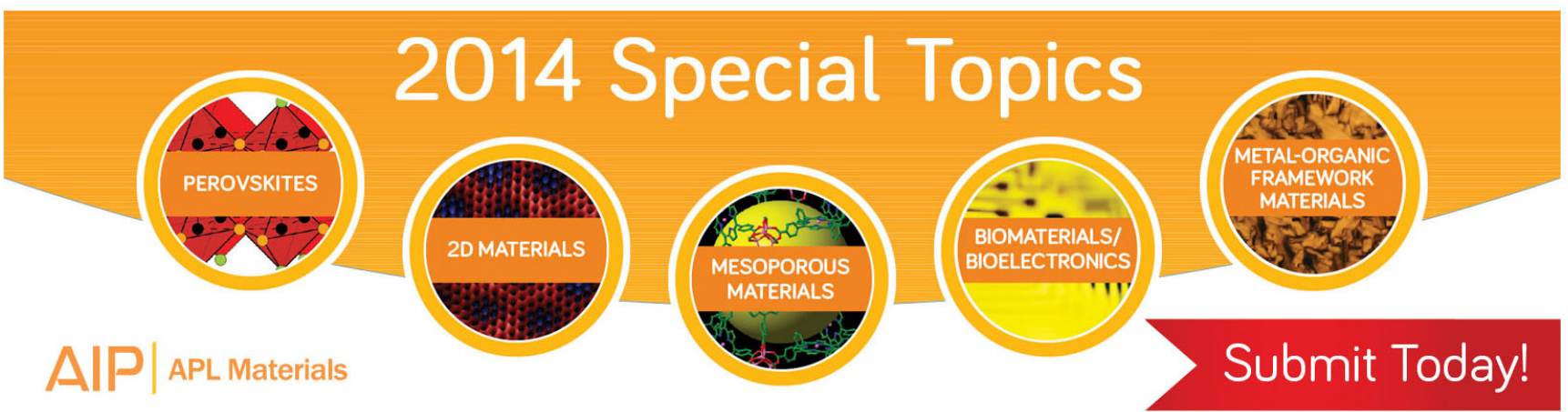




\title{
A versatile photoelectron spectrometer for pressures up to $\mathbf{3 0}$ mbar
}

\author{
Susanna K. Eriksson, ${ }^{1}$ Maria Hahlin, ${ }^{2}$ Juhan Matthias Kahk, ${ }^{3}$ Ignacio J. Villar-Garcia, ${ }^{3}$ \\ Matthew J. Webb, ${ }^{4}$ Helena Grennberg, ${ }^{4}$ Rositza Yakimova, ${ }^{5}$ Håkan Rensmo, ${ }^{2}$ \\ Kristina Edström, ${ }^{1}$ Anders Hagfeldt, ${ }^{1}$ Hans Siegbahn, ${ }^{2}$ Mårten O. M. Edwards, ${ }^{6}$ \\ Patrik G. Karlsson, ${ }^{6}$ Klas Backlund, ${ }^{6}$ John Åhlund, ${ }^{6, a)}$ and David J. Payne ${ }^{3}$ \\ ${ }^{1}$ Department of Chemistry-Ångström, Uppsala University, Box 523, 75120 Uppsala, Sweden \\ ${ }^{2}$ Department of Physics and Astronomy, Uppsala University, Box 516, 75120 Uppsala, Sweden \\ ${ }^{3}$ Department of Materials, Imperial College London, Exhibition Road, London SW7 2AZ, United Kingdom \\ ${ }^{4}$ Department of Chemistry-BMC, Uppsala University, Box 576, 75123 Uppsala, Sweden \\ ${ }^{5}$ Department of Physics, Chemistry, and Biology, Linköping University, SE-581 83 Linköping, Sweden \\ ${ }^{6}$ VG Scienta AB, Box 15120, 75015 Uppsala, Sweden
}

(Received 11 April 2014; accepted 8 July 2014; published online 30 July 2014)

\begin{abstract}
High-pressure photoelectron spectroscopy is a rapidly developing technique with applications in a wide range of fields ranging from fundamental surface science and catalysis to energy materials, environmental science, and biology. At present the majority of the high-pressure photoelectron spectrometers are situated at synchrotron end stations, but recently a small number of laboratory-based setups have also emerged. In this paper we discuss the design and performance of a new laboratory based high pressure photoelectron spectrometer equipped with an $\mathrm{Al} \mathrm{K} \alpha \mathrm{X}$-ray anode and a hemispherical electron energy analyzer combined with a differentially pumped electrostatic lens. The instrument is demonstrated to be capable of measuring core level spectra at pressures up to 30 mbar. Moreover, valence band spectra of a silver sample as well as a carbon-coated surface (graphene) recorded under a 2 mbar nitrogen atmosphere are presented, demonstrating the versatility of this laboratory-based spectrometer. (C) 2014 AIP Publishing LLC. [http://dx.doi.org/10.1063/1.4890665]
\end{abstract}

\section{INTRODUCTION}

$\mathrm{X}$-ray Photoelectron spectroscopy (XPS) is one of the most valuable and widely used techniques for the characterization of the electronic structure and composition of materials, with a tuneable level of surface sensitivity. In common usage, XPS is performed under conditions of high or ultrahigh vacuum. Yet, since the early days of the technique methods have been developed to also allow the acquisition of spectra under higher pressures. The first "non-high-vacuum" XPS experiments on liquid phase samples were performed by Kai and Hans Siegbahn. ${ }^{1}$ Their instrument for measurements of liquids used a liquid beam. A similar design finds current use in the state-of-the-art liquid-jet photoemission experiments. ${ }^{2-4}$ Further designs were based on a "wetted wire" 5 or a rotating metal $\operatorname{disk}^{6}$ for transporting the liquid into the analysis chamber. The majority of today's typical high-pressure photoelectron spectrometers are designed primarily for the study of solid surfaces under a high pressure (HP) gas atmosphere, and incorporate a small-volume "high-pressure" cell ${ }^{7}$ or a backfilled analysis chamber ${ }^{8}$ as described in more detail in Ref. 9. This concept can be traced back to the pioneering work of Roberts in the late 1970s. ${ }^{10}$ His system, later commercialized under the name VG ESCALAB HP, incorporated a differentially pumped high-pressure cell and was capable of performing XPS measurements at pressures from UHV to 1 mbar. ${ }^{11}$ A significant limitation was the relatively low transmission of photoelectrons through the analyzer when operating at high pressure, which could not be overcome even with differential pumping, due to the diverging nature of the pho-

\footnotetext{
a) Author to whom correspondence should be addressed. Electronic mail: john.ahlund@vgscienta.com
}

toelectrons after emission from the solid. The development of a 2nd generation hemispherical analyzer incorporating electrostatic lensing within the different pumping stages to maximize photoelectron transmission has brought the High Pressure X-ray Photoelectron Spectroscopy (HPXPS) technique once again to the forefront of photoelectron spectroscopy. ${ }^{12}$ The technique has been primarily developed at synchrotron radiation sources utilizing a high photon flux. ${ }^{13,14}$

While the use of synchrotron light sources has obvious advantages, it severely restricts the widespread use of the technique due to the limited number of synchrotron end stations with HPXPS capabilities. ${ }^{9,15}$ Increasing availability is one of the key challenges if HPXPS is to be utilized to its full potential in a wide variety of subject areas, and indeed in the recent years there has been renewed interest in the development of laboratory-based HPXPS instruments utilizing differentially pumped analyzers and focused X-ray sources. ${ }^{9}, 16,17$ In processes ranging from heterogeneous catalysis ${ }^{18}$ and electrochemical reactions in devices such as fuel cells, ${ }^{19}$ solar cells, ${ }^{20,21}$ and batteries ${ }^{22-24}$ to environmental chemistry ${ }^{25}$ and biomaterials ${ }^{26}$ HPXPS is a fast-growing and valuable addition to the suite of advanced characterization techniques available to the scientific community. Below we report on a design allowing for home laboratory-based HPXPS measurements at pressures above the room temperature vapour-pressure of water and with a flexible configuration for efficient studies of a large variety of systems.

\section{SYSTEM DESIGN}

The basic design of the present HPXPS set-up is outlined in Figure 1. It consists of an X-ray source (Scienta MX 


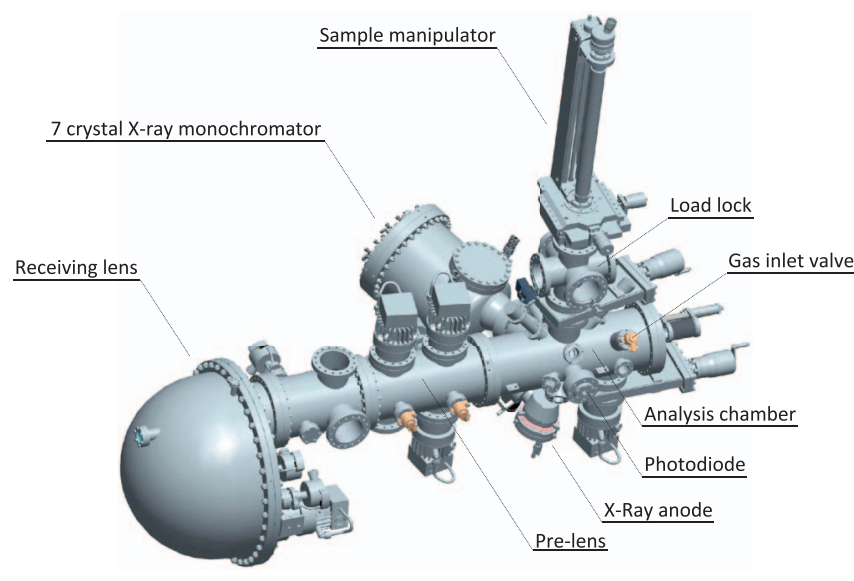

FIG. 1. Schematic 3D drawing of the instrument set up.

$650 \mathrm{HP}$ ), a stainless steel analysis chamber with a $\mu$-metal liner, load lock chamber, and an electron energy analyzer (Scienta R4000 HiPP-2). The X-ray monochromator is mounted at an angle of $62.5^{\circ}$ with respect to the symmetry axis of the analyzer pre-lens. The sample is mounted on a sample stage connected to a VG Scienta Transax four-axis manipulator with $\mathrm{x}, \mathrm{y}$, and $\mathrm{z}$ translations and polar rotation.

During operation it is essential that all high voltage elements are kept under a high vacuum, while a considerable gas pressure may be present in the neighbouring analysis chamber. To achieve this, the X-ray source has been separated from the analysis chamber by a reinforced aluminium coated window, and two additional pumping stages have been introduced between the analysis chamber and the electron energy analyzer. The outermost front cone of the differential pumping setup protruding into the analysis chamber is exchangeable. This allows the size of the first aperture to be varied to optimize the setup for the particular pressure range of interest.

The physical layout of the differential pumping stages in the analyzer is shown in Figure 2(a). To maintain vacuum in the entire system during high vacuum operation, as well as steep pressure gradients during high-pressure operation, there are a total of seven turbomolecular pumps supported by three scroll pumps. There are two turbo pumps connected to the 1 st stage of the differential pumping system, and one each to the load lock, the analysis chamber, the X-ray monochromator, the 2nd stage of the differential pumping system, and the hemispherical analyzer (3rd stage). The load lock above the analysis chamber is pumped by a Varian Turbo-V 81-AG type turbo pump (pumping rate for nitrogen $77 \mathrm{l} / \mathrm{s}$ ); all other turbo pumps are of type Varian Turbo-V 301 Navigator (pumping rate for nitrogen $250 \mathrm{l} / \mathrm{s}$ ). The scroll pumps are divided as follows: one backs the turbo pumps on the load lock, monochromator, and analysis chamber where four additional valves allow each part to be pumped individually; one backs those on the 1st differential pumping stage; and one backs those on the 2 nd pumping stage and the 3 rd pumping stage. The base pressure of the system is in the $10^{-8}$ mbar range.

For high-pressure work, the turbo pumps on the load lock and analysis chamber are turned off and the corresponding valves to backing lines are closed, and the entire analysis chamber and the load lock are gas filled from a needle valve. (a)

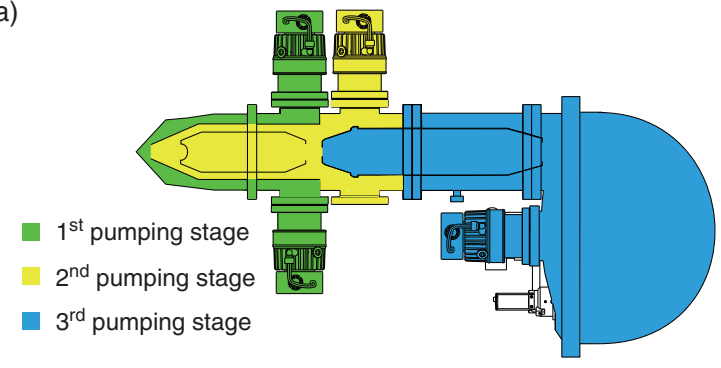

(b)

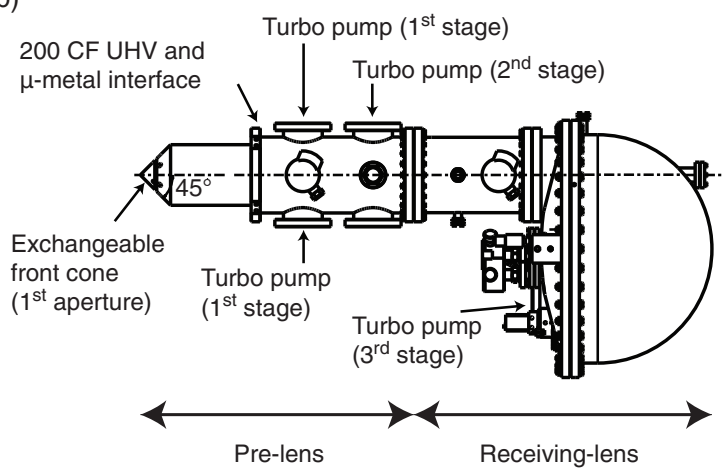

FIG. 2. (a) Schematic illustration of the different pumping zones of the highpressure analyzer used for the system. The pre-lens contains two pumping zones (green and yellow), whereas the receiving lens is pumped by one turbo pumped located on the baseplate of the hemisphere. (b) Schematic drawing of the Scienta R4000 HiPP-2 analyzer. The pre- and receiving lenses are indicated, as well as connection point of the analyzer and the analysis chamber, the exchangeable front cone and pump flanges.

The primary advantage, beyond simplicity, of back-filling the entire analysis chamber during high-pressure work, as opposed to the alternative design approach where a dedicated high-pressure cell is used, is that it places fewer geometrical constraints on the types of samples that can be studied under high pressure, e.g., the sample can be tilted several tens of degrees. The principle of optimizing the setup for studies of a range of different types of samples, as well as the possible use of other equipment in the analysis chamber, has also been taken into account in the design of the $\mathrm{X}$-ray source and the analyzer, as detailed in Secs. II A and II B.

The pressure in the analysis chamber is probed by either a piezo transducer or one of two baratron transducers (all from MKS). However, the true pressure at the sample surface will be affected by the distance to the analyzer aperture into the 1st analyzer pumping stage. It is estimated that the relative difference between the analysis chamber pressure and the true sample pressure is less than $10 \%$, provided the sample distance, i.e., the distance between the sample surface and the analyzer front cone, is equal to or greater than the diameter of the cone, as per the analysis by Ogletree et al. ${ }^{27}$ A procedure for accurately adjusting the sample distance from the front cone aperture is crucial for a proper sample pressure treatment. The point of zero distance between the sample and the analyzer cone may be determined by driving the sample towards the cone when the analysis chamber is under high pressure and monitoring the pressure in the first pumping stage of the pre-lens. When the sample makes contact the pressure in the first pumping stage falls abruptly. Alternatively, if the 
sample is X-ray illuminated, the point of zero distance may be determined by observing when the analyzer count rate drops to zero. This method is better if the samples are soft or sensitive. Subsequently, the sample is positioned at a distance equal to the cone diameter of the used analyzer front cone using the micrometre precision stage of the manipulator, giving a sample pressure that is about $90 \%$ of the analysis chamber pressure.

Additional pressure readings are available from an ion gauge in the X-ray monochromator and a cold cathode in the 1 st pumping stage of the analyzer pre-lens. There is a bypass line between the analysis chamber and the X-ray monochromator. The bypass line is kept closed during high-pressure operation to maintain high vacuum in the $\mathrm{X}$-ray source whereas it must be open during venting and pump down of the analysis chamber to avoid breakage of the thin X-ray window.

\section{A. X-ray source}

The X-rays are produced in an $\mathrm{Al} \mathrm{K} \alpha$ source (Scienta MX650 HP) with an enhanced focus relative to the standard Scienta MX650 model. The Scienta MX650 HP source consists of an aluminium anode unit, a quartz crystal monochromator, and an X-ray window for maintaining vacuum in the X-ray source while a considerable gas pressure may be present in the analysis chamber. The reinforced aluminium coated window is $44 \mathrm{~mm}$ in diameter and in the order of 1 $\mu \mathrm{m}$ thick and situated at a distance of $102 \mathrm{~mm}$ from the sample focus. The durability of the X-ray window has been tested to withstand a pressure difference up to at least 50 mbar and no change in the pressure in the X-ray monochromator is observed, even when pressures in the tens of mbar range are present in the analysis chamber, proving that no measurable leakage occurs through the window. The metallic coating of the window ensures that it does not get charged during operation. The large size of the window matches the solid angle of the radiation cone from the seven crystals in the monochromator. The water-cooled aluminium anode is bombarded with a finely focused electron beam, and the Bragg reflections from the monochromator crystals are focused onto the sample in the analysis chamber. The maximum electron-beam operation power of the source is $200 \mathrm{~W}$.

The monochromator comprises seven closely packed toroidally shaped $\alpha$-quartz crystals mounted in a $650 \mathrm{~mm}$ diameter Rowland circle configuration. The diameter of each crystal is $76 \mathrm{~mm}$. The crystals are kept at an elevated temperature of $55^{\circ} \mathrm{C}$, ensuring that the crystal lattice spacing is not affected by temperature variations in the laboratory. Each crystal can be individually adjusted in energy and position using a set of three screws that are manually controlled from the outside of the monochromator. The peak energy of the monochromized X-rays is $1486.6 \mathrm{eV}$, and the energy full width at half maximum (FWHM) is $168 \mathrm{meV}^{28}$

\section{B. Analyzer design}

A schematic of the Scienta R4000 HiPP-2 analyzer is shown in Figure 2(b). Conceptually it can be divided into two distinct parts: the pre-lens and the receiving lens. The photoelectrons first travel a short distance in the analysis chamber, then pass through the pre-lens, and finally enter the receiving lens, where their kinetic energies are resolved. The analyzer uses the Scienta R4000 hemispherical electron energy analyzer as the receiving lens. ${ }^{29}$ The analyzer is connected to the analysis chamber via a $200 \mathrm{CF}$ flange, ensuring a good UHV connection. The connection is located $300 \mathrm{~mm}$ from the focus point of the analyzer, which is also the connection point for the $\mu$-metal liner of the analysis chamber to the analyzer. The front of the analyzer is cone shaped with a $45^{\circ}$ angle with respect to the symmetry axis. This design allows for sample illumination angles near the magic incidence angle of $\sim 55^{\circ}$ and the usage of monochromators with a large solid angle radiation cone. Combined with the position of the high-pressure window of the X-ray source, it also gives a large degree of freedom for sample manipulation, which facilitates studies of physically large samples, such as reaction vessels, electronic circuitry, etc., or the usage of auxiliary equipment in the analysis chamber.

The analyzer can be run in two distinct modes of operation. The overall intensity of the photoelectron signal is maximized and spatial information is retained in transmission mode, while the emission angle of the photoelectrons is measured in angular mode.

The receiving lens, based on the conventional Scienta $\mathrm{R} 4000$ model, is a $200 \mathrm{~mm}$ mean radius analyzer equipped with 9 interchangeable slits ranging from $0.1 \mathrm{~mm}$ to $4 \mathrm{~mm}$ in width. The electron optics consists of a set of electrostatic lens elements and can be operated using pass energies between 2 and $200 \mathrm{eV}$. For the experiments performed here pass energies of $20 \mathrm{eV}$ for gas phase analyzer resolution tests and 100 and $200 \mathrm{eV}$ for XPS measurements are used. It features a twodimensional detector that consists of a FireWire CCD camera and a $\varnothing 40 \mathrm{~mm}$ Multi-Channel Plate (MCP) detector. The simultaneous energy range detected is $8 \%$ of the pass energy and the geometrical magnification of the electron beam at the detector in transmission mode is $\times 5$, which ensures a high information rate as described in Ref. 29.

The pre-lens is a differentially pumped electrostatic lens system that consists of a set of entrance apertures and an exit aperture. The first entrance aperture is located on the apex of the exchangeable cone, allowing the diameter of this aperture to be varied. In the experiments reported in this paper, first apertures with a diameter of $0.3 \mathrm{~mm}, 0.5 \mathrm{~mm}$, and $0.8 \mathrm{~mm}$ are used. In the text they will be mentioned as $0.3 \mathrm{~mm}$ cone, $0.5 \mathrm{~mm}$ cone, and $0.8 \mathrm{~mm}$ cone. The front cone restricts the field of view of the analyzer. The field of view for a particular cone is proportional to the area of the aperture. A second aperture is located $10 \mathrm{~mm}$ downstream from the first. The short distance between the first and second apertures assures a rapid decrease of pressure within the pre-lens, according to the design philosophy in Refs. 7 and 14. In standard operation the second aperture is on ground potential and thereby geometrically limiting the acceptance angle of the pre-lens, which is $\pm 15^{\circ}$ from the optical axis. A third aperture is located at the exit of the pre-lens. At this position the electrostatic pre-lens is set to produce an intermediate slightly magnified imaging point of the sample. The length of the third aperture, being in 
the non-energy dispersive direction of the hemispherical analyzer, is chosen such that it does not restrict the field of view or angular divergence of the intermediate image along this direction. The width of the pre-lens exit aperture, being in the energy dispersive direction of the hemispherical analyzer, has a smaller dimension selected to match the divergence properties of the receiving lens in combination with the widths of the most commonly used slits of the hemispherical analyzer. As the R4000 hemispherical analyzer uses slit pairs, ${ }^{29}$ the energy resolution is usually determined by the selected pass energy and analyzer entrance slit, i.e., in principle independent of the retardation ratio or lens mode operation. However, for the configuration presented in this report the width of the prelens exit aperture is too small to match the largest selectable analyzer slits. This results in that the largest slits produce "too good" energy resolution at the expense of that the information rate does not increase much when changing from an intermediate slit to a large one. In the present work, the size of this exit aperture has been optimized for high transmission using a pass energy of 100-200 eV, resulting in an overall full width half maximum line width of less than $0.9 \mathrm{eV}$ for the $\mathrm{Ag} 3 d$ core levels. It is noteworthy that when higher resolution is desirable, it has been shown that in order to maximize transmission for a given resolution, it is favourable to operate a hemispherical analyzer with a combination of a small slit and a higher pass energy rather than with a large slit and a low pass energy. ${ }^{29}$

\section{SYSTEM SPECIFICATIONS}

\section{A. X-ray source performance}

Using a polycrystalline silver foil as the sample and the $0.5 \mathrm{~mm}$ cone, the position of the anode as well as the tilting and positions of the monochromator crystals were optimized to maximize the intensity of the $\mathrm{Ag} 3 d$ photoemission signal. This initial X-ray source alignment was kept fixed throughout the measurements presented in the present paper.

The flux from the X-ray source and the transmission of the high-pressure window were determined using a $10 \mathrm{~mm}$ $\times 10 \mathrm{~mm}$ photodiode (AXUV100, Optodiode, USA) and a current amplifier (DLPCA-200, Femto, Germany). A truncated analyzer front cone was used and the photodiode was positioned $20 \mathrm{~mm}$ downstream from the exit focus point of the $\mathrm{X}$-ray source, which is also the focus point of the spectrometer, monochromator along the monochromator optical axis (see Figure 3). In this geometry the Bragg reflected $\mathrm{Al} \mathrm{K \alpha}$ radiation from all seven crystals impinges on the active area of the photodiode. Measuring the flux with the high-pressure window mounted, the window transmission could be determined as $76 \% \pm 2 \%$. This value is quite high taking into account the large dimensions of the window. The window in this laboratory based instrument needs to be much larger than those of the windows used for synchrotron radiation sources as it needs to match the solid angle of the radiation cone in order to maximize transmission. The high-pressure window acts as an efficient radiation blocker for unwanted radiation in the soft X-ray regime and lower energy regimes, and there

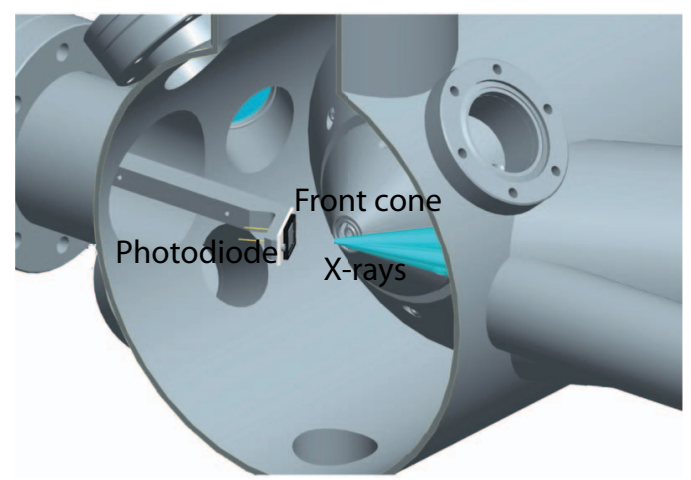

FIG. 3. A schematic picture of the photodiode measurements where the truncated front cone, the photodiode, and the X-ray beam are marked.

is essentially no detectable photodiode background when the window is mounted. It is worth noting that the total flux is dependent upon the acceleration voltage of the electron beam, even if the power is kept constant. Throughout the measurements a power of $200 \mathrm{~W}$ was used in combination with an acceleration voltage of $12 \mathrm{kV}$ in the present paper. Under these conditions the flux is $3.7 \times 10^{10}$ photons s $^{-1}$.

The profile of the X-ray beam from the monochromator was investigated by hole throughput and knife edge measurements. A thin metallic knife-edge blade was mounted on the sample holder such that it could be controlled by the 4-way manipulator. A circular hole with a diameter of $1.0 \mathrm{~mm}$ was laser cut in the blade. The position and tilting of the blade were optimized for maximum radiation throughput through the $\varnothing 1.0 \mathrm{~mm}$ hole. At the optimal position the hole is normal to the monochromator optical axis and centred in the beam focal waist. The maximum throughput was determined to be $41 \%$. This value corresponds to an effective FWHM of $1.15 \mathrm{~mm}$, if the beam profile is assumed to be Gaussian and symmetric around the optical axis. However, as will be seen below, the beam profile is neither symmetric nor a pure Gaussian.

Next, knife-edge widths of the beam profile were determined in both the horizontal and vertical directions by moving the knife-edge into the beam by the manipulator and recording the photodiode signal. For a Gaussian profile the width between $12 \%$ and $88 \%$ of the initial signal intensity corresponds directly to the FWHM of the beam profile. The 12/88 knife-edge widths were determined as $1.3 \mathrm{~mm}$ and $2.2 \mathrm{~mm}$ in the vertical and horizontal directions, respectively. The disagreement between the hole throughput and the 12/88 widths indicates that the profile is non-Gaussian. This is further supported from measurements of 10/90 widths and 20/80 widths, which point at a profile with a higher central density than the Gaussian distribution.

The horizontal and vertical 12/88 widths of the projected $\mathrm{X}$-ray spot in the analyzer sample plane were determined as $4.7 \mathrm{~mm}$ and $1.4 \mathrm{~mm}$. The vertical width is effectively unchanged whereas the horizontal profile is elongated due to the $62.5^{\circ}$ projection in the present system. To compare, recalculation to the magic angle $54.7^{\circ}$, which commonly is used in XPS systems, gives a horizontal width of $3.8 \mathrm{~mm}$. The attenuation of the X-ray beam due to scattering on gas atoms was 


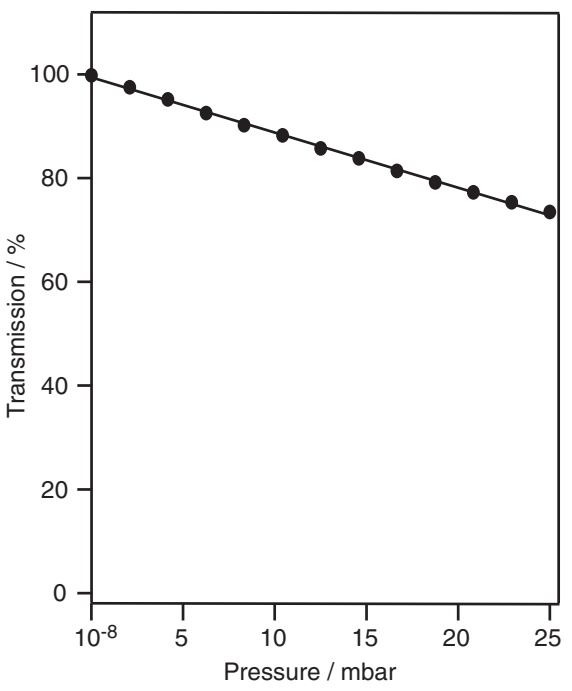

FIG. 4. Flux in the analyzer focus point versus nitrogen pressure in the analysis chamber.

investigated by measuring the X-ray flux, by the photodiode, as a function of the nitrogen gas pressure in the analysis chamber. However, as we are interested in the flux at the analyzer focal point, the measured exponential decline to the photodiode position (122 $\mathrm{mm}$ from the $\mathrm{X}$-ray window) is recalculated to the analyzer focal point (102 $\mathrm{mm}$ from the X-ray window). The resulting focal point flux data and a fitted line are displayed versus the analysis chamber pressure in Figure 4. It can be seen that the transmission is higher than $90 \%$ up to approximately $10 \mathrm{mbar}$ and that it still is as high as $74 \%$ at 25 mbar, meaning that the X-ray source with the highpressure window is a suitable excitation tool for high-pressure XPS studies in large-volume chambers all the way up to the water vapour pressure ( $\sim 25 \mathrm{mbar})$ at room temperature.

\section{B. Pumping efficiency of the differential pumping stages}

Ensuring low pressures in the differential pumping stages is crucial in order to maximize electron transmission to the analyzer. Moreover, pressure should be low enough to ensure the safe operation of the turbo pumps connected to these stages. High pressures could also produce electric discharges from the analyzer high-voltage elements. Hence, an efficient pumping of the differential pumping stages is critical for analysis of samples at high pressures.

The first performed test concentrated on checking that there were no other internal leaks in the analyzer other than through the first aperture. With a pressurised analysis chamber (atmospheric pressure), the pressure in the analyzer was measured while using a blinded first aperture. No pressure fluctuations were observed in the analyzer, so it can be concluded that the analysis chamber is pumped solely through the first aperture, which ensures an effective use of the differential pumping stages.

During operation at high pressures, there is a continuous gas leakage from the analysis chamber into the first differential pumping stage through the first aperture. These gases

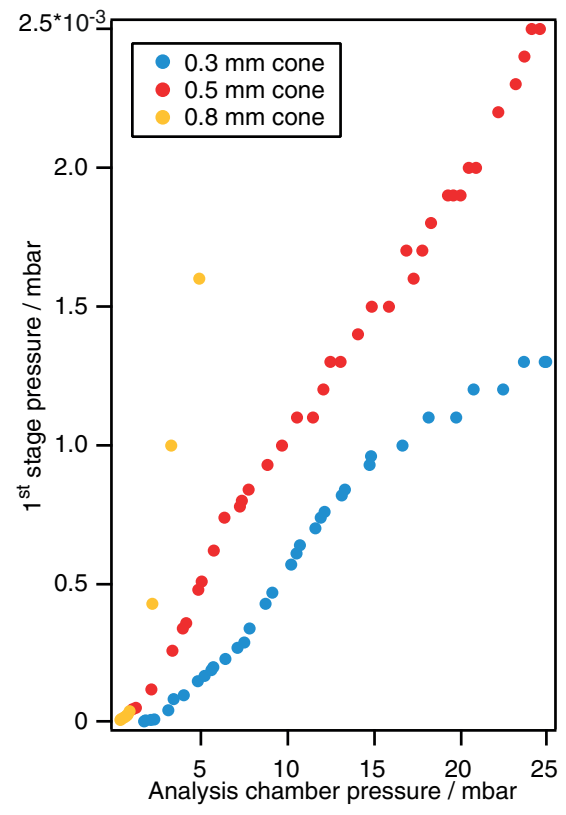

FIG. 5. First pumping stage pressure versus analysis chamber pressure (nitrogen atmosphere) for three different cone diameters.

are pumped away from the first differential pumping stage through two turbo pumps directly connected to the first pumping stage and through the second aperture. The second and third differential pumping stages are pumped down by one turbo pump each. The main way to control the pressure in the first pumping stage is by varying the size of the cone diameter. To test the pumping efficiency of the different pumping stages with different first apertures sizes, the pressures of the first and second differential pumping stages of the system were monitored as the pressure in the analysis chamber was increased by slowly filling it with nitrogen gas. During these tests the second differential pumping stage was measured to be in the low $10^{-5}$ mbar range for all cones and all analysis chamber pressures tested. This ensures high electron transmission from the second aperture onwards in all analysis pressure conditions.

Figure 5 shows the pressure in the first differential pumping versus the analysis chamber pressure for the three cones available. It can be seen that, for the same pressure in the analysis chamber, there are larger pressure differences between the analysis chamber and the first differential pumping stage for the smaller cone diameter sizes. For example, for a pressure of 5 mbar in the analysis chamber, the pressure in the first differential pumping stage with the $0.5 \mathrm{~mm}$ cone $\left(5.1 \times 10^{-4} \mathrm{mbar}\right)$ was four times higher than the pressure measured with the $0.3 \mathrm{~mm}$ cone $\left(1.5 \times 10^{-4}\right.$ mbar $)$ and it was one order of magnitude higher for the $0.8 \mathrm{~mm}$ cone $\left(1.6 \times 10^{-3}\right.$ mbar). It can be clearly seen in Figure 5 that as the pressure increases in the analysis chamber the pressure in the first differential pumping stage increases more steeply for larger cone apertures, which consequently can be operated in a more limited pressure regime. For example, a linear extrapolation of the $0.8 \mathrm{~mm}$ cone pressure relation to an analysis chamber pressure of $25 \mathrm{mbar}$ would give a first pumping stage pressure of approximately $1 \times 10^{-2}$ mbar. 

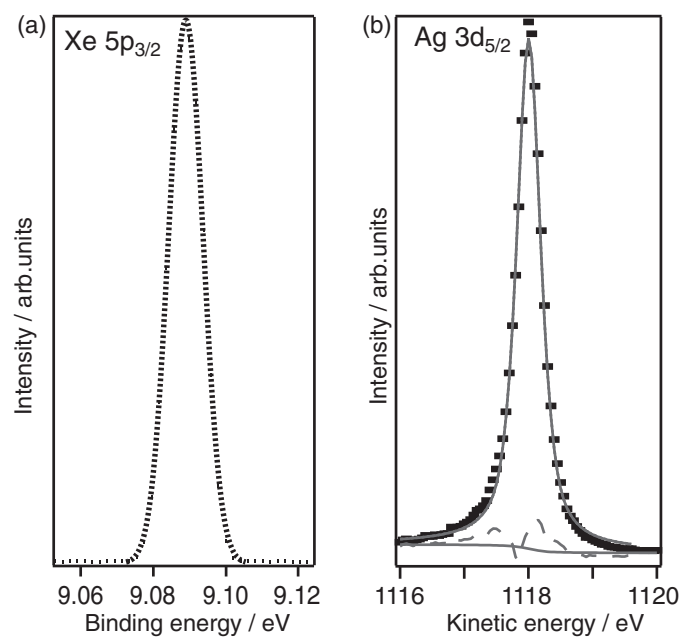

FIG. 6. (a) Xe $5 p_{3 / 2}$ high-resolution spectrum of xenon gas showing an analyzer energy resolution of $10.6 \mathrm{meV}$. The spectrum was acquired at high vacuum and at room temperature, with a $0.2 \mathrm{~mm}$ slit width and $20 \mathrm{eV}$ pass energy. The excitation source was a $21.2 \mathrm{eV} \mathrm{He} \mathrm{I} \mathrm{UV-light.} \mathrm{For} \mathrm{this} \mathrm{measure-}$ ment the front cone was replaced with a truncated cone with a wide opening in order to fit the gas cell. (b) $\mathrm{Ag} 3 d_{5 / 2}$ high-resolution spectrum of a clean silver sample showing a total resolution of $450 \mathrm{meV}$. The spectrum was acquired at high vacuum and at room temperature, with a $0.3 \mathrm{~mm}$ slit width, $0.5 \mathrm{~mm}$ cone, $100 \mathrm{eV}$ pass energy, $0.05 \mathrm{eV}$ step size, and $5 \mathrm{~min}$ acquisition time. The photon energy was $1486.6 \mathrm{eV}$ (Al K $\alpha$ X-rays).

\section{Energy resolution and intensity in high vacuum}

The resolution of the Scienta R4000 HiPP-2 analyzer was tested by measuring the Xe $5 p_{3 / 2}$ high-resolution spectrum (see Figure 6(a)) of Xe gas (N48, 99.998\% from Air Liquid, Scienta GC50 gas cell) excited by monochromated $21.2 \mathrm{eV}$ He I UV-light from a Scienta VUV5000 light source. The analyzer resolution was calculated to be $10.6 \mathrm{meV}$ after de-convolution of the Doppler broadening of $3.25 \mathrm{meV}$ and the light source broadening of $1.2 \mathrm{meV} .{ }^{30}$ This value is very close to the theoretical resolution limit of $10.0 \mathrm{meV},{ }^{31}$ showing the high resolving capacity of the analyzer.

To test the energy resolution and intensity of the instrument, room temperature high-resolution spectra of $\mathrm{Ag}$ $3 d$ were measured. A clean silver foil sample $(99.95 \%$, K. A. Rasmussen, Norway) was used in accordance with the ISO 15470/2004 standard: "Surface chemical analysis - X-ray photoelectron spectroscopy - Description of selected instrumental performance parameters." 32 The Ag sample was scraped with a scalpel and introduced in the system load lock just before pumping down the load lock. No O $1 \mathrm{~s}$ signal was detected in the $\mathrm{O} 1 s$ high-resolution spectrum and the $\mathrm{C} 1 s$ contribution was less than $5 \%$ of the $\mathrm{Ag} 3 d$ signal. The FWHM and intensities were obtained, after subtracting a Shirley background, ${ }^{33}$ by fitting the recorded peaks with a Voigt function that accounts for the Gaussian instrumental broadening and temperature effects as well as the Lorentzian life time broadening contribution. All Ag $3 d$ spectra were measured using the photon energy $1486.6 \mathrm{eV}$ (Al K $\alpha$ X-rays). The energy step was $0.05 \mathrm{eV}$ and the measurement time was 7 min.

The FWHM of the Ag $3 d_{5 / 2}$ peak (see Figure 6(b)) was measured to be $450 \mathrm{meV}$, which is in accordance with the product specifications $(<500 \mathrm{meV}$, Scienta MX650, Scienta HiPP-2) and a good match to the theoretical resolution (413 meV) under the measurement conditions. This theoretical resolution was calculated using the empirical expression given by Olivero et $a .^{34}$ as the product of the convolution of the Gaussian instrumental contributions from the analyzer (75 meV for a 200 mean radius hemispherical analyzer at a pass energy of $100 \mathrm{eV}$ and a slit width of $0.3 \mathrm{~mm}$ ) and the X-ray source (168 meV, see Ref. 28), the Gaussian temperature broadening ( $90 \mathrm{meV}$ for measurements at room temperature $\left.{ }^{35,36}\right)$, and the Lorentzian lifetime broadening of the photoinduced core hole $(310 \mathrm{meV}$ for the $\mathrm{Ag}$ $3 d_{5 / 2}$ transition $^{37}$ ). The total resolution of $450 \mathrm{meV}$ is dominated by the lifetime broadening contribution of the $\mathrm{Ag} 3 d_{5 / 2}$ state, and the instrumental contribution to the resolution is calculated to be $205 \mathrm{meV}$. In Figure 7, the experimental and theoretical resolution of the $\mathrm{Ag} 3 d_{5 / 2}$ peak is plotted as a function of analyzer slit width for $100 \mathrm{eV}$ and $200 \mathrm{eV}$ pass energies for all three cones. For small slits the resolution matches the theoretical values, while for larger slits the resolution is far below the theoretically calculated values. The deviation between the experimental and theoretical values sets on at a slit width of approximately $1 \mathrm{~mm}$. Below this break point the resolution is set by the analyzer slit width, whereas the resolution is determined by the size of the exit entrance aperture of the pre-lens above the break point. The intensity also flattens out similarly to the resolution when the analyzer slit width reaches $1 \mathrm{~mm}$ as displayed in Figure 8 . As expected the $0.8 \mathrm{~mm}$ cone gives the highest intensities and $200 \mathrm{eV}$ pass energy gives higher intensities compared to $100 \mathrm{eV}$, considering that the number of sweeps, step time, dwell time, and kinetic energy interval is the same for both pass energies (resulting in a difference in total recording time, as indicated in Figures 7 and 8).

\section{Sensitivity and energy resolution at elevated pressures}

One of the main features of this system is the ability to choose between different first apertures by changing the front cone. To investigate the optimum cone diameter size at every pressure range, $\mathrm{Ag} 3 d_{5 / 2}$ high-resolution spectra were acquired at pressures ranging from high vacuum up to several mbar using the different cones available. The distance from the sample to the front cone aperture was adjusted to one aperture diameter, i.e., $0.3,0.5$, and $0.8 \mathrm{~mm}$, respectively. Figure 9 displays the attenuation curve (signal intensity versus pressure) for the three different cones. The reason for the intensity variation at high vacuum conditions relates to the fact that the spot size of the X-ray beam on the sample is larger than any of the available cone diameter sizes (approximately 1.4 $\times 4.7 \mathrm{~mm}$ ). Therefore, at high vacuum conditions the cone defines the field of view for the analyzer which is the main factor controlling the intensity of the signal. As the pressure increases the signal intensity becomes increasingly affected by the electron losses in the gas phase. Therefore, the distance the electrons have to travel at high pressure (the same as the cone diameter in our operating conditions) will have a higher influence for higher analysis pressures. Going back to Figure 9 it can be seen, as expected, that the highest signal 

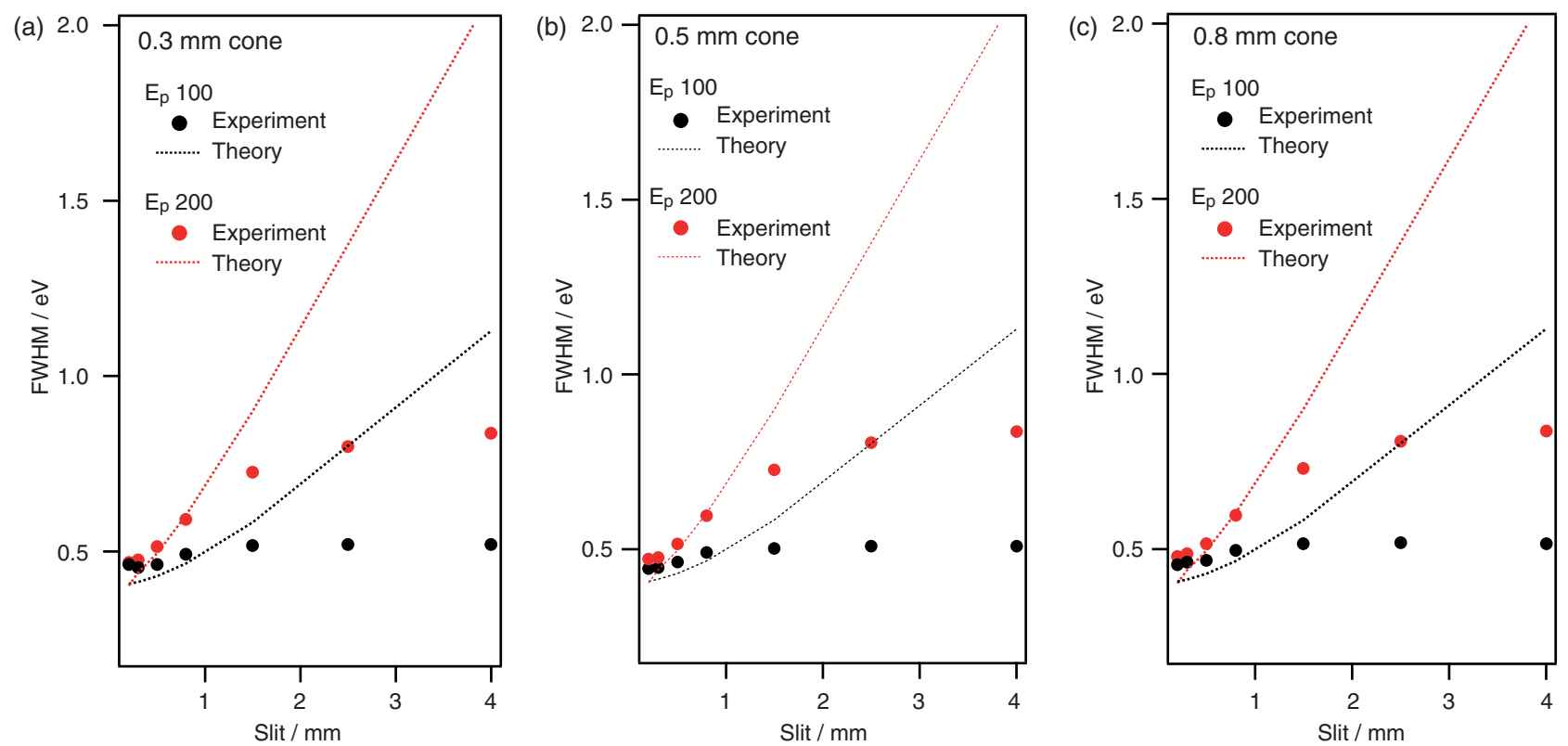

FIG. 7. (a) Experimental and theoretical FWHM of the Ag $3 d_{5 / 2}$ peak versus slit size for 100 and $200 \mathrm{eV}$ pass energies for different cone diameter sizes: $0.3 \mathrm{~mm}$ (a), $0.5 \mathrm{~mm}$ (b), and $0.8 \mathrm{~mm}$ (c). The spectra were acquired at high vacuum and at room temperature with a $0.05 \mathrm{eV}$ step size and a dwell time of $1 \mathrm{~s}$. The spectra were recorded between $1112 \mathrm{eV}$ and $1118 \mathrm{eV}$ kinetic energy for both $100 \mathrm{eV}$ and $200 \mathrm{eV}$ pass energy. The spectra are normalized to one sweep with $7.5 \mathrm{~min}$ acquisition time for $100 \mathrm{eV}$ pass energy and $13 \mathrm{~min}$ acquisition time for $200 \mathrm{eV}$ pass energy.

intensities are obtained for the $\mathrm{Ag} 3 d_{5 / 2}$ peaks acquired at high vacuum and the highest of all is found for the largest cone diameter $(0.8 \mathrm{~mm})$. As the pressure increases the loss in intensity is more rapid for the larger cones. After drawing linear guide lines for each cone diameter size, it can be observed that the 0.8 and $0.5 \mathrm{~mm}$ cone attenuation curves intersect at approximately 2 mbar and the 0.5 and $0.3 \mathrm{~mm}$ cone attenua-

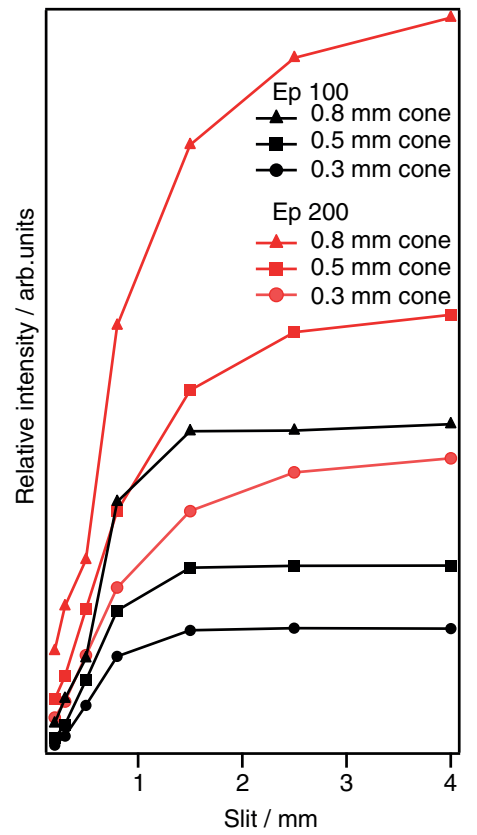

FIG. 8. Intensity versus slit size for the different cones and pass energies. The spectra were acquired at high vacuum and at room temperature with a $0.05 \mathrm{eV}$ step size and a dwell time of $1 \mathrm{~s}$. The spectra were recorded between $1112 \mathrm{eV}$ and $1118 \mathrm{eV}$ kinetic energy for both $100 \mathrm{eV}$ and $200 \mathrm{eV}$ pass energy. The spectra are normalized to one sweep with $7.5 \mathrm{~min}$ acquisition time for $100 \mathrm{eV}$ pass energy and $13 \mathrm{~min}$ acquisition time for $200 \mathrm{eV}$ pass energy. tion curves intersect at approximately 4 mbar. The $\mathrm{Ag} 3 d_{5 / 2}$ high-resolution spectra at pressures close to these intersections for the different cones are shown in Figure 10. It can be concluded that the $0.5 \mathrm{~mm}$ cone offers a superior performance only within the 2-4 mbar pressure range, while the $0.8 \mathrm{~mm}$ cone will provide with higher intensities below 2 mbar and the $0.3 \mathrm{~mm}$ cone at pressures over 4 mbar. In Figure 11(a) the normalized $\mathrm{Ag} 3 d_{5 / 2}$ spectra acquired under high vacuum and at approximately 3 and 16 mbar nitrogen pressures are shown. No change in energy resolution or line shape is observed with increasing pressure. Furthermore, the

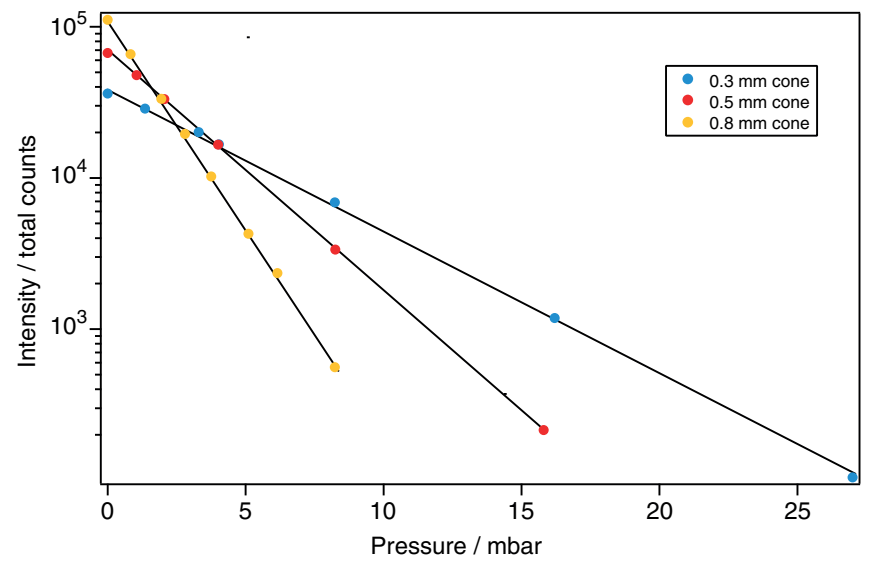

FIG. 9. Attenuation curves: logarithm of the $\mathrm{Ag} 3 d_{5 / 2}$ peak intensity versus pressure (nitrogen atmosphere) for different cone diameters. 0.8 and $0.5 \mathrm{~mm}$ cone attenuation curves intersect at approximately $2 \mathrm{mbar}$ and the 0.5 and $0.3 \mathrm{~mm}$ cone attenuation curves intersect at approximately 4 mbar. All spectra are acquired from a clean silver sample at room temperature, with a $4 \mathrm{~mm}$ slit width, $200 \mathrm{eV}$ pass energy, $0.05 \mathrm{eV}$ step size. The acquisition time varied from approximately $7 \mathrm{~min}$ for high vacuum spectra to $30 \mathrm{~min}$ for high-pressure spectra. The intensities are normalized versus one sweep. 

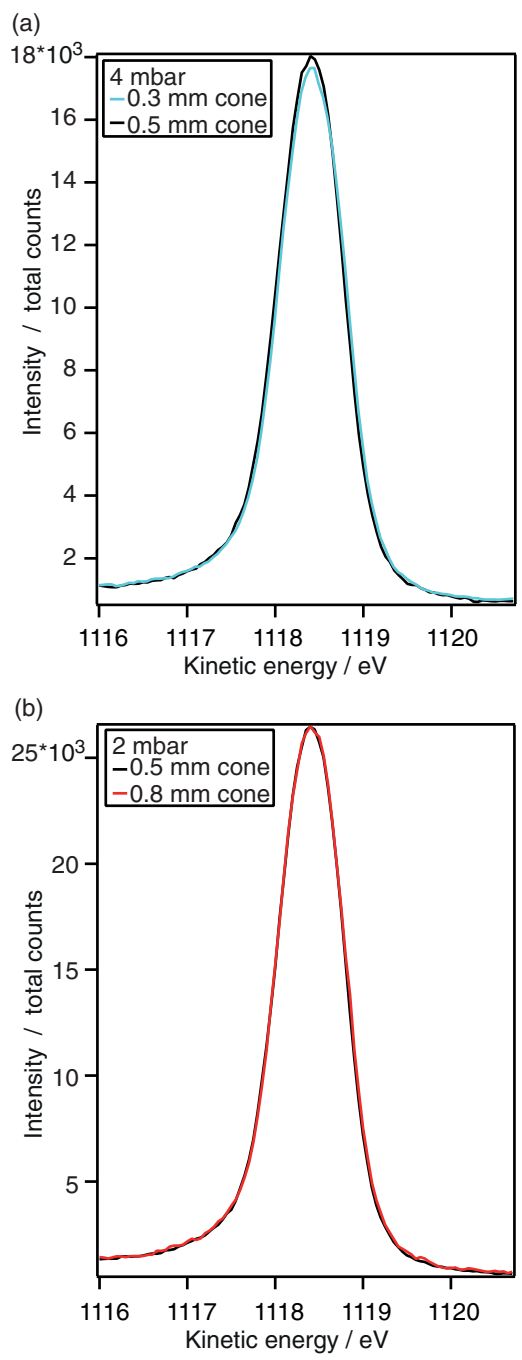

FIG. 10. (a) $\mathrm{Ag} 3 d_{5 / 2}$ core level spectra, acquired at different pressures (nitrogen atmosphere) and cone diameters showing: (a) The $\mathrm{Ag} 3 d_{5 / 2}$ peak intensities at 4 mbar for the 0.3 and $0.5 \mathrm{~mm}$ cones. (b) The $\mathrm{Ag} 3 d_{5 / 2}$ peak intensities at $2 \mathrm{mbar}$ for the 0.5 and $0.8 \mathrm{~mm}$ cones. All spectra were acquired from a clean silver sample at room temperature, with a $4 \mathrm{~mm}$ slit width, $200 \mathrm{eV}$ pass energy, $0.05 \mathrm{eV}$ step size. Acquisition times were maximum $30 \mathrm{~min}$.

FWHMs for all the spectra acquired for the data in Figures 10 and 11 are also the same $(0.84 \mathrm{eV})$. It can be concluded that the energy resolution for this system is independent of the pressure (pressure range in this study: high vacuum to 30 mbar) and cone diameter size $(0.3 \mathrm{~mm}, 0.5 \mathrm{~mm}$, and $0.8 \mathrm{~mm})$.

To test the limits of the system, we have acquired an Ag $3 d_{5 / 2}$ core level spectrum that was measured at 30 mbar as displayed in Figure 11(b). To the best of our knowledge, this represents the highest pressure PES spectrum recorded with a laboratory-based system that has been reported in the scientific literature. ${ }^{16}$ The FWHM of the $\mathrm{Ag} 3 d_{5 / 2}$ peak at 30 mbar is also $0.84 \mathrm{eV}$. All $\mathrm{Ag} 3 d$ spectra were measured using the photon energy $1486.6 \mathrm{eV}$ (Al K $\alpha$ X-rays) and the spectra displayed in Figures 10 and 11 were all measured using pass energy of $200 \mathrm{eV}$. The energy step was $0.05 \mathrm{eV}$ and the measurement time was 7-30 $\mathrm{min}$ for pressures up to 16 mbar. The acquisition time was $4.5 \mathrm{~h}$ and the energy step $0.07 \mathrm{eV}$ for the spectrum measured at $30 \mathrm{mbar}$.
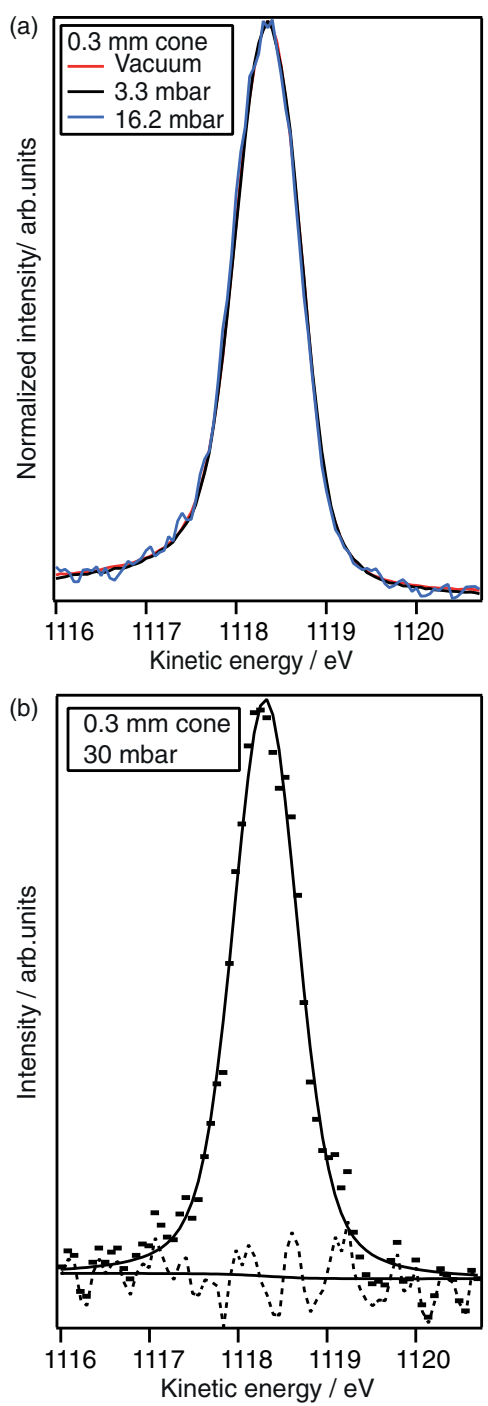

FIG. 11. (a) Area normalized Ag $3 d_{5 / 2}$ high-resolution spectra of a clean silver sample at high vacuum, $3.3 \mathrm{mbar}$ and $16.2 \mathrm{mbar}$ (nitrogen atmosphere) acquired with the $0.3 \mathrm{~mm}$ cone. All peaks exhibit the same FWHM values. (b) $\mathrm{Ag} 3 d_{5 / 2}$ spectrum of a clean silver sample under a 30 mbar nitrogen atmosphere. The spectrum was acquired at room temperature; with a $4 \mathrm{~mm}$ slit width, a $0.3 \mathrm{~mm}$ cone, $200 \mathrm{eV}$ pass energy, $0.07 \mathrm{eV}$ step size, and $4.5 \mathrm{~h}$ acquisition time. The FWHM of the peak is $0.84 \mathrm{eV}$.

We have also noticed that the 0.8 cone and larger X-ray spot is good for radiation sensitive materials since a larger area of the sample will be irradiated but with lower intensity compared to a smaller spot. This opens up possibilities to measure sensitive organic and biologic samples.

\section{E. Wide energy range performance}

To demonstrate the high performance of the analyzer over a broad energy range we display the survey spectra of an aspresented (dirty) silver sample and the same sample after it was scraped with a scalpel (clean sample). The clean Ag sample spectra were recorded under vacuum conditions as well as under 1 mbar $\mathrm{N}_{2}$ pressure. The spectra show good statistics over the full kinetic energy range, including the MVV Auger transition of $\mathrm{Ag}$ at around $350 \mathrm{eV}$ kinetic energy. A close-up of this feature is shown by the inset in Figure 12. To further 


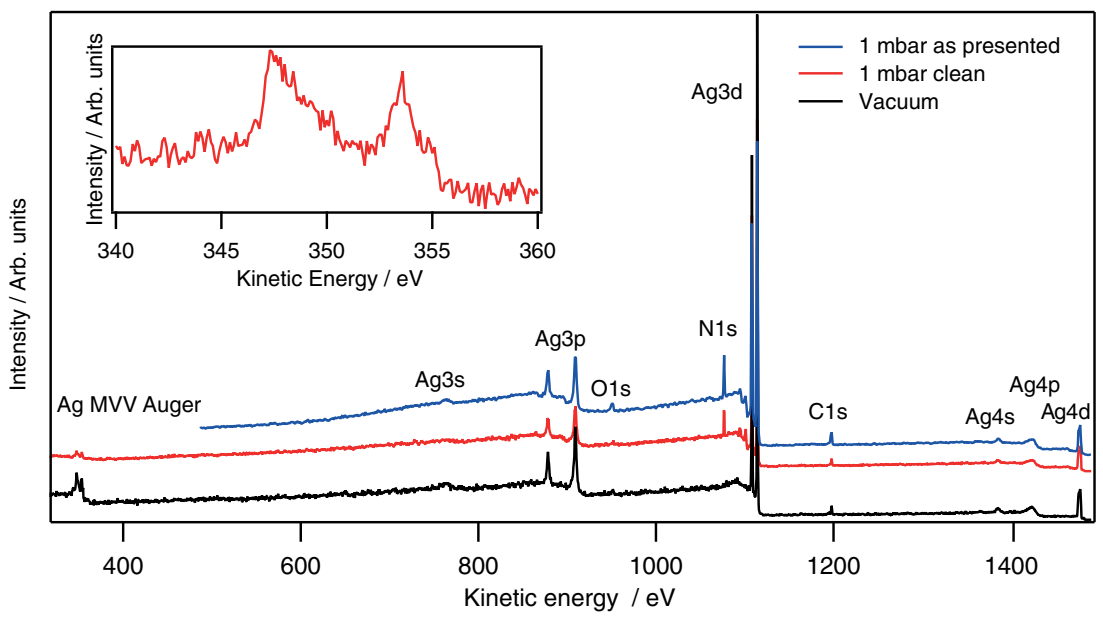

FIG. 12. Survey spectra of a clean Ag sample recorded with 1 mbar $\mathrm{N}_{2}$ atmosphere. Included is also a survey spectrum from a dirty surface to show the capabilities of measuring small impurities. As an inset the MVV Auger transition is showed to illustrate the capabilities at lower kinetic energies.

illustrate the capabilities of the system to detect and measure low concentration species an Ag sample with expected surface contamination is also included in Figure 12. Here the oxygen contamination around at $530 \mathrm{eV}$ binding energy and the carbon contamination at $285 \mathrm{eV}$ are clearly visible. This clearly shows the capability of measuring photoelectrons with a very low surface concentration. The survey spectrum was collected using the $0.8 \mathrm{~mm}$ front cone, $0.5 \mathrm{eV}$ step size, $100 \mathrm{eV}$ pass energy, and $4 \mathrm{~mm}$ slit for the clean sample and $0.8 \mathrm{~mm}$ slit for the dirty sample. The total measurement time was $1 \mathrm{~h}$ for the vacuum sample and $1.5 \mathrm{~h}$ for the clean $1 \mathrm{mbar}$ sample and $2 \mathrm{~h}$ for the oxidized sample. The MVV Auger transition (inset in Figure 12) was recorded using $0.8 \mathrm{~mm}$ front cone, $0.1 \mathrm{eV}$ step size, $100 \mathrm{eV}$ pass energy, and $4 \mathrm{~mm}$ slit, and the spectrum acquisition time was about $30 \mathrm{~min}$.

\section{F. Outlook: High pressure surface science}

For an $\mathrm{Al} \mathrm{K} \alpha$ excitation source, low binding energy photoionization such as those occurring for the valence band and C $1 s$ electrons exhibit the lowest photoionization cross sections across the accessible spectral energy range. To demonstrate the system capabilities, in this section we present valence band spectra for a traditional silver sample as well as a carbon coated surface (graphene) acquired with the system at pressures up to $2 \mathrm{mbar}$. These valence band spectra are, to the best of our knowledge, the first valence band spectra acquired at pressures above 1 mbar in a laboratory based system. ${ }^{10,17}$ We also present a $\mathrm{C} 1 s$ spectrum of graphene acquired at both high vacuum and high pressures. For the graphene measurements the 0.8 cone was used, the slit was $0.8 \mathrm{~mm}$, and the step $0.05 \mathrm{eV}$. The acquisition times were $45 \mathrm{~min}$ for $\mathrm{C} 1 s$ and $6 \mathrm{~h}$ for the valence bands. The Ag valence band was recorded using the same settings as for graphene but with an acquisition time of $3.5 \mathrm{~h}$. The photon energy was $1486.6 \mathrm{eV}$ and the pass energy was $200 \mathrm{eV}$. The Ag valence and the graphene sample were energy calibrated using the $\mathrm{N}_{2}$-gas signal. ${ }^{38}$ The $\mathrm{N}_{2}$-gas signal is expected to be shifted depending on the solid materials work function ${ }^{39}$ but here we calibrate on the gas signal for simplicity.
The normalized Ag valence band spectra obtained at high vacuum and at 2 mbar nitrogen atmosphere are shown in Figure 13. It is observed that also in this energy range the resolution for the valence band spectra is the same both at high vacuum and at 2 mbar. Furthermore, the recorded spectrum at 2 mbar offers details directly related to the atmosphere within the system, i.e., the nitrogen valence band is observed as the difference between the spectra at high vacuum and at high pressure. ${ }^{38,40} \mathrm{We}$ also display the $\mathrm{N}_{2}$-signal measured on the pure gas and it is as seen in Figure 12 identical with the difference spectrum.

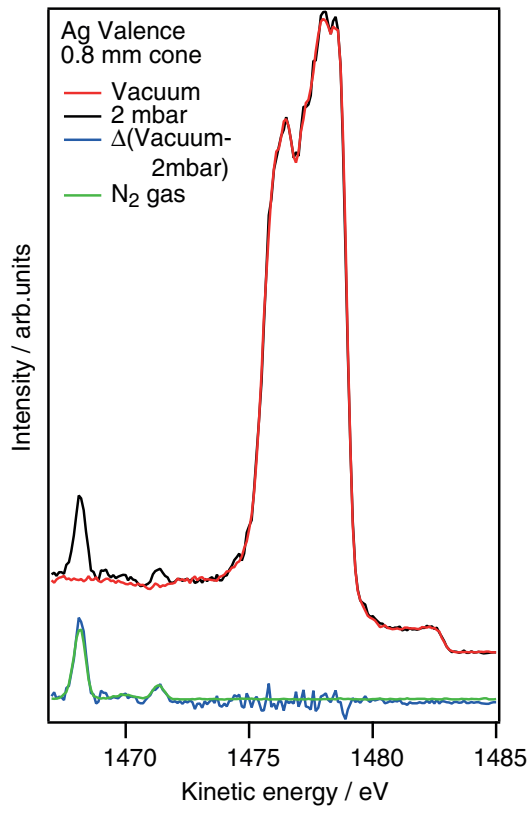

FIG. 13. Normalized Ag valence band of a clean silver sample at high vacuum and at 2 mbar of nitrogen atmosphere and the difference between the two spectra. The differential trace exhibits the characteristic features of the nitrogen valence band spectrum as seen in the $\mathrm{N}_{2}$-gas valence spectrum acquired without a solid sample present in the chamber and in literature. ${ }^{38}$ The spectra were acquired at room temperature, with a $0.8 \mathrm{~mm}$ slit width, $0.8 \mathrm{~mm}$ cone, $200 \mathrm{eV}$ pass energy, $0.05 \mathrm{eV}$ step size. The acquisition times were $3.5 \mathrm{~h}$. 
In order to further probe the capabilities of the instrument a more scientifically and industrially relevant material was investigated. Graphene is one of the most intensely studied materials of the 21 st century. ${ }^{41}$ The growth of graphene and its intentional modification by various means have been studied extensively by photoemission techniques in UHV. ${ }^{42}$ High-pressure studies can offer a more realistic view of these processes when investigated under ambient atmospheric conditions. Characterization of such a system involves both core level and valence band measurements of a few monolayer of carbon material on a substrate. A well-resolved $\mathrm{C} 1 s$ spectrum
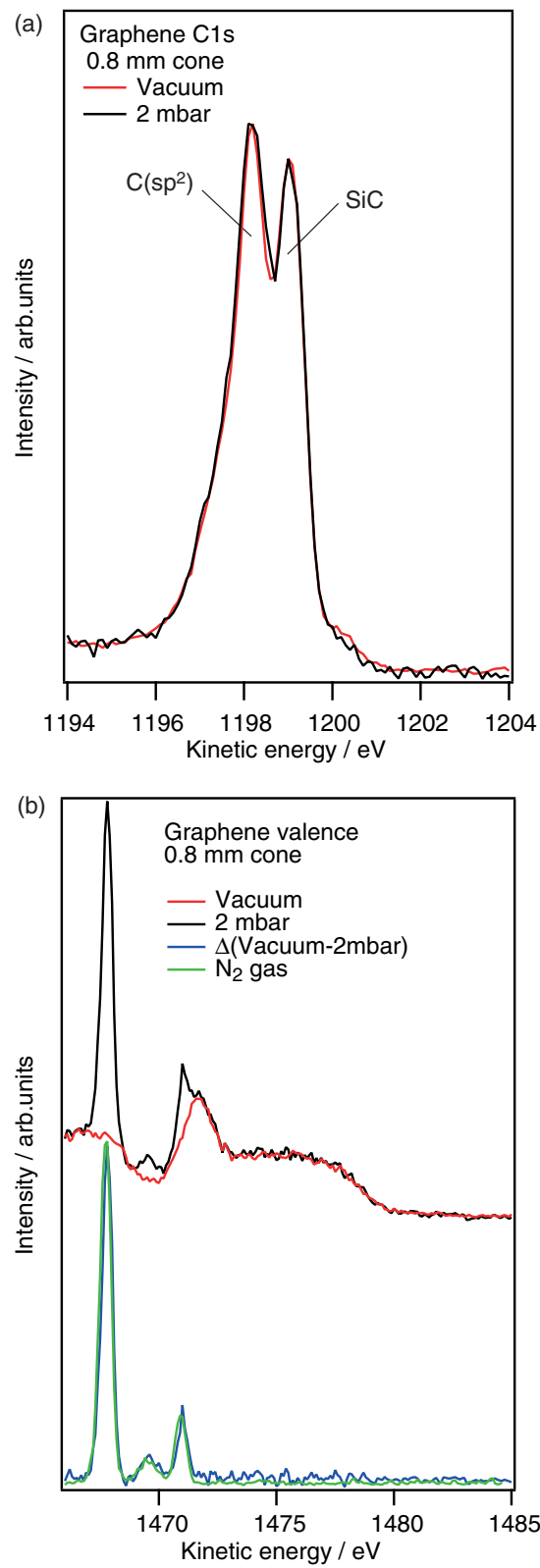

FIG. 14. (a) Normalized C $1 s$ high resolution spectra at high vacuum and at 2 mbar of nitrogen atmosphere of bi-layer graphene on silicon carbide. ${ }^{38}$ (b) Normalized valence band spectrum at high vacuum and at 2 mbar of nitrogen atmosphere of the same sample and the difference between the two spectra. The differential trace exhibits the characteristic features of the nitrogen valence band spectrum as seen in the $\mathrm{N}_{2}$ gas valence spectrum acquired without a solid sample present in the chamber and in literature. ${ }^{38}$ The spectra were acquired at room temperature, with a $0.8 \mathrm{~mm}$ slit width, $0.8 \mathrm{~mm}$ cone, $200 \mathrm{eV}$ pass energy, $0.05 \mathrm{eV}$ step size. The acquisition times were $45 \mathrm{~min}$ for the core levels and $6 \mathrm{~h}$ for the valence bands. is essential in the characterization of the formed graphene layers and its interactions with the substrate. To show the quality of the sample as well as the performance of the instrument, we present the $\mathrm{C} 1 s$ high-resolution spectra in Figure 14(a) recorded for bi-layer graphene on silicon carbide ${ }^{43,44}$ in high vacuum and at 2 mbar nitrogen atmosphere. The signal is composed of two partially resolved peaks and a small shoulder at the low kinetic part of the signal. The first partially resolved peak at the low kinetic energy side of the spectrum $(\sim 1198 \mathrm{eV})$ is characteristic of the $s p^{2}$ hybridised carbon atoms in the graphene framework and the small shoulder at even lower energies has been related to the carbon buffer layer. ${ }^{45}$ The second partially resolved peak at the high kinetic part of the signal $(\sim 1199 \mathrm{eV})$ originates from the silicon carbide bulk of the sample. Figure 14(b) shows the recorded valence band measurements for the same graphene sample under high vacuum and at 2 mbar nitrogen atmosphere. Again, the nitrogen valence band is observed as the difference between the spectra at high vacuum and high pressure. ${ }^{38}$ These spectra were recorded within a time frame of only 4-6 h, showing the potential of surface sensitive HPXPS studies on applied systems.

\section{CONCLUSIONS}

A high-pressure photoelectron spectroscopy system has been developed for use at home laboratories. The system is capable of providing high quality data at pressures up to $30 \mathrm{mbar}$. The excellent performance of the instrument is based on the combination of a Scienta MX 650 HP X-ray source and a Scienta R4000 HiPP-2 analyzer. The X-ray source is separated from the main chamber by an X-ray window of high Xray transmittance $(74 \%)$ and that can withstand pressures of at least 50 mbar. The analyzer combines three differentially pumped stages and electromagnetic lenses to maximize electron transmission and detection. The first aperture of the analyzer can be varied by using front cones of variable diameter $(0.3,0.5$, and $0.8 \mathrm{~mm})$ allowing the optimization of instrument performance (maximizing signal intensity) within different pressure regimes. The design of the first aperture of the analyzer as a cone protruding into the analysis chamber and the large degree of freedom and space in the analysis chamber will enable the analysis of a wide variety of sample types and shapes such as powders, films, large area wafers as well as biological materials. This instrument has allowed us to acquire valence band spectra at high pressures in a laboratory. The valence band spectra for silver and graphene samples at 2 mbar are of high quality, exhibit very good signal to noise ratios, and high-energy resolution (FWHMs are equal to the same measurements taken at high vacuum). The possibility of measuring routine high resolution PES in a home laboratory at high pressures makes this technique available to a wider range of users and is expected to significantly contribute to the development of many areas of science.

\section{ACKNOWLEDGMENTS}

This system was developed at VG Scienta AB with funding from Swedish Governmental Agency for Innovation 
Systems (VINNOVA). S.K.E., K.E., M.H., H.R., H.S., and A.H. would like to acknowledge The Swedish Energy Agency and the Swedish Research Council (2012-4681). R.Y., M.J.W., and H.G. are grateful for financial support from the Swedish Research Council (621-2010-4799) and the Knut \& Alice Wallenberg Foundation. D.J.P. acknowledges support from the Royal Society (UF100105). J.M.K. acknowledges support from the EPSRC for a Doctoral Prize Studentship. D.J.P and I.J.V.G acknowledge support from the EPSRC (EP/K004913/1 and EP/J021199/1).

${ }^{1}$ K. Siegbahn, "From X-ray to electron spectroscopy and new trends," J. Electron Spectrosc. Relat. Phenom. 51, 11-36 (1990).

${ }^{2}$ M. Faubel, B. Steiner, and J. P. Toennies, "Photoelectron spectroscopy of liquid water, some alcohols, and pure nonane in free micro jets," J. Chem. Phys. 106(22), 9013-9031 (1997).

${ }^{3}$ B. Winter and M. Faubel, "Photoemission from liquid aqueous solutions," Chem. Rev. 106(4), 1176-1211 (2006).

${ }^{4}$ M. A. Brown et al., "A new endstation at the Swiss Light Source for ultraviolet photoelectron spectroscopy, X-ray photoelectron spectroscopy, and X-ray absorption spectroscopy measurements of liquid solutions," Rev. Sci. Instrum. 84(7), 073904 (2013).

${ }^{5} \mathrm{H}$. Fellnerfeldegg et al., "ESCA applied to liquids. IV. A wire system for ESCA measurements on liquids," J. Electron Spectrosc. Relat. Phenom. 7(5), 421-428 (1975).

${ }^{6} \mathrm{H}$. Siegbahn, S. Svensson, and M. Lundholm, "A new method for ESCA studies of liquid-phase samples," J. Electron Spectrosc. Relat. Phenom. 24(2), 205-213 (1981).

${ }^{7} \mathrm{~F}$. Mangolini et al., "Angle-resolved environmental X-ray photoelectron spectroscopy: A new laboratory setup for photoemission studies at pressures up to 0.4 Torr,” Rev. Sci. Instrum. 83(9), 093112 (2012).

${ }^{8}$ J. Schnadt et al., "The new ambient-pressure X-ray photoelectron spectroscopy instrument at MAX-lab," J. Synchrotron Radiat. 19, 701-704 (2012).

${ }^{9}$ D. E. Starr et al., "Investigation of solid/vapor interfaces using ambient pressure X-ray photoelectron spectroscopy," Chem. Soc. Rev. 42(13), 5833-5857 (2013).

${ }^{10}$ R. W. Joyner, M. W. Roberts, and K. Yates, "A "high-pressure" electron spectrometer for surface studies," Surf. Sci. 87(2), 501-509 (1979).

${ }^{11}$ R. W. Joyner and M. W. Roberts, "Study of the adsorption of oxygen on silver at high-pressure by electron-spectroscopy," Chem. Phys. Lett. 60(3), 459-462 (1979).

${ }^{12}$ D. F. Ogletree et al., "Photoelectron spectroscopy under ambient pressure and temperature conditions," Nucl. Instrum. Methods Phys. Res., Sect. A 601(1-2), 151-160 (2009).

${ }^{13} \mathrm{H}$. Bluhm et al., "In situ x-ray photoelectron spectroscopy studies of gassolid interfaces at near-ambient conditions," MRS Bull. 32(12), 1022-1030 (2007).

${ }^{14}$ M. E. Grass et al., "New ambient pressure photoemission endstation at Advanced Light Source beamline 9.3.2," Rev. Sci. Instrum. 81(5), 053106 (2010).

${ }^{15} \mathrm{H}$. Bluhm, "Photoelectron spectroscopy of surfaces under humid conditions," J. Electron Spectrosc. Relat. Phenom. 177(2-3), 71-84 (2010).

${ }^{16} \mathrm{~F}$. Tao, "Design of an in-house ambient pressure AP-XPS using a bench-top $\mathrm{X}$-ray source and the surface chemistry of ceria under reaction conditions," Chem. Commun. 48(32), 3812-3814 (2012).

${ }^{17}$ K. Roy, C. P. Vinod, and C. S. Gopinath, "Design and performance aspects of a custom-built ambient pressure photoelectron spectrometer toward bridging the pressure gap: Oxidation of $\mathrm{Cu}, \mathrm{Ag}$, and $\mathrm{Au}$ surfaces at 1 mbar O2 pressure," J. Phys. Chem. C 117(9), 4717-4726 (2013).

${ }^{18}$ S. Kaya et al., "Ambient-pressure photoelectron spectroscopy for heterogeneous catalysis and electrochemistry," Catal. Today 205, 101-105 (2013).

${ }^{19} \mathrm{C}$. J. Zhang et al., "Measuring fundamental properties in operating solid oxide electrochemical cells by using in situ X-ray photoelectron spectroscopy,” Nat. Mater. 9(11), 944-949 (2010).
${ }^{20}$ A. Hagfeldt et al., "Dye-sensitized solar cells," Chem. Rev. 110(11), 6595$6663(2010)$

${ }^{21}$ I. Josefsson et al., "Collective hydrogen-bond dynamics dictates the electronic structure of aqueous I-3(-)," Phys. Chem. Chem. Phys. 15(46), 20189-20196 (2013).

${ }^{22}$ Y.-C. Lu et al., "In situ ambient pressure X-ray photoelectron spectroscopy studies of lithium-oxygen redox reactions," Sci. Rep. 2, 715 (2012).

${ }^{23} \mathrm{~S}$. Malmgren et al., "Consequences of air exposure on the lithiated graphite SEI," Electrochim. Acta 105, 83-91 (2013).

${ }^{24}$ B. Philippe et al., "Nanosilicon electrodes for lithium-ion batteries: Interfacial mechanisms studied by hard and soft X-ray photoelectron spectroscopy," Chem. Mater. 24(6), 1107-1115 (2012).

${ }^{25}$ H. Bluhm and H. C. Siegmann, "Surface science with aerosols," Surf. Sci. 603(10-12), 1969-1978 (2009).

${ }^{26} \mathrm{G}$. Ketteler et al., "In situ photoelectron spectroscopy study of water adsorption on model biomaterial surfaces," J. Phys.: Condens. Matter. 20(18), 184024 (2008).

${ }^{27}$ D. F. Ogletree et al., "A differentially pumped electrostatic lens system for photoemission studies in the millibar range," Rev. Sci. Instrum. 73(11), 3872-3877 (2002).

${ }^{28} \mathrm{H}$. Fellner-Feldegg, Computer Simulation of the Concave Grazing Incidence Monochromator (Uppsala University, 1974).

${ }^{29} \mathrm{~N}$. Martensson et al., "A very high-resolution electron spectrometer," J. Electron Spectrosc. Relat. Phenom. 70(2), 117-128 (1994).

${ }^{30} \mathrm{P}$. Baltzer et al., "Resolution and signal-to-background enhancement in gas-phase electron-spectroscopy,” Rev. Sci. Instrum. 64(8), 2179-2189 (1993).

${ }^{31}$ Practical Surface Analysis by Auger and X-ray Photoelectron Spectroscopy, edited by D. Briggs and M. P. Seah (John Wiley and Sons, 1983)

${ }^{32}$ ISO, "Surface chemical analysis-X-ray photoelectron spectroscopyDescription of selected instrumental performance parameters," ISO 15470:2004, Geneva.

${ }^{33}$ D. A. Shirley, "High-resolution X-ray photoemission spectrum of valence bands of gold," Phys. Rev. B 5(12), 4709 (1972).

${ }^{34}$ J. J. Olivero and R. L. Longbothum, "Empirical fits to voigt linewidth—Brief review," J. Quant. Spectrosc. Radiat. Transfer 17(2), 233-236 (1977).

${ }^{35}$ S. Hüfner, Photoelectron Spectroscopy-Principles and Applications (Springer, Berlin, 2003).

${ }^{36} \mathrm{~S}$. Mahl et al., "Characterisation of the VG ESCALAB instrumental broadening functions by XPS measurements at the Fermi edge of silver," J. Electron Spectrosc. Relat. Phenom. 85(3), 197-203 (1997).

${ }^{37}$ J. L. Campbell and T. Papp, "Widths of the atomic K-N7 levels," At. Data Nucl. Data Tables 77, 1-56 (2001).

${ }^{38}$ K. Kimura, Handbook of HeI Photoelectron Spectra of Fundamental Organic Molecules (Japan Scientific Societies Press, 1981), p. 268.

${ }^{39} \mathrm{~S}$. Axnanda et al., "Direct work function measurement by gas phase photoelectron spectroscopy and its application on PbS nanoparticles," Nano Lett. 13(12), 6176-6182 (2013).

${ }^{40} \mathrm{U}$. Gelius et al., "A high resolution ESCA instrument with X-ray monochromator for gases and solids," J. Electron Spectrosc. Relat. Phenom. 2(5), 405-434 (1973).

${ }^{41}$ K. S. Novoselov et al., "A roadmap for graphene," Nature (London) 490(7419), 192-200 (2012).

${ }^{42} \mathrm{~K}$. V. Emtsev et al., "Interaction, growth, and ordering of epitaxial graphene on $\mathrm{SiC}\{0001\}$ surfaces: A comparative photoelectron spectroscopy study," Phys. Rev. B 77(15), 155303 (2008).

${ }^{43} \mathrm{C}$. Virojanadara et al., "Homogeneous large-area graphene layer growth on $6 \mathrm{H}-\mathrm{SiC}(0001)$," Phys. Rev. B: Condens. Matter Mater. Phys. 78(24), 245403-1-245403-6 (2008)

${ }^{44} \mathrm{~K}$. V. Emtsev et al., "Towards wafer-size graphene layers by atmospheric pressure graphitization of silicon carbide," Nat. Mater. 8(3), 203-207 (2009).

${ }^{45}$ C. Riedl, C. Coletti, and U. Starke, "Structural and electronic properties of epitaxial graphene on $\mathrm{SiC}(0001)$ : A review of growth, characterization, transfer doping and hydrogen intercalation,” J. Phys. D: Appl. Phys. 43(37), 374009-1-374009-17 (2010). 